\title{
Mechanism of Action of Eosin-Methylene Blue Agar in the Differentiation of Escherichia coli and Enterobacter aerogenes
}

\author{
R. S. HORVATH and M. E. ROPP
}

\begin{abstract}
Department of Biology, Bowling Green State University, Bowling Green, Ohio 43403
\end{abstract}
\begin{abstract}
The mechanism involved in the action of eosin-methylene blue agar (EMB) in differentiating Escherichia coli from Enterobacter aerogenes was investigated using prototrophic strains of these organisms and a $\mathrm{Lac}^{-}$auxotroph, E. coli $\mathrm{X}-961$. The final $\mathrm{pH}$ of the EMB agar resulting from growth of these organisms at the expense of lactose was shown to play a critical role in the differential action. An EMB complex, which formed under acidic conditions, appeared to be involved in the differential action of this medium. The molecular weight of the complex indicated an eosin to methylene blue ratio of 1 to 1 . Ultraviolet and infrared spectral data indicated the occurrence of an amide bond between the dyes when complexing occurred. On this basis, a mechanism for complexing of eosin and methylene blue, under acidic conditions, was proposed.
\end{abstract}

The use of eosin-methylene blue (EMB) agar as a differential medium for the colon-typhoiddysentery group has become well established since its introduction by Holt-Harris and Teague (2) in 1916. The medium contained the dyes eosin and methylene blue, which yielded a sharp distinction between lactose- and nonlactose-fermenting organisms.

Two years later, Levine (3) employed a modification of the original EMB medium and concluded that the modified EMB could be used to differentiate lactose fermenters from non-lactose fermenters.

Although the medium had been used extensively since its introduction, the mechanism of its differential action was not known. Wilson (6), in a review of differential staining by mixtures of eosin and methylene blue, called attention to a dye complex which formed under acidic conditions. It was proposed that such a complex might be responsible for the differential action of EMB agar.

Wynne, Rode, and Hayward (7) assumed that the differential action of EMB was a direct function of the acid produced during fermentation. Pathogens belonging to the genera Salmonella and Shigella were compared with the nonpathogens Escherichia coli and two Enterobacter species, and it was concluded that the pathogens could not ferment lactose to produce a sufficiently low $\mathrm{pH}$ to result in colored colonies on EMB, whereas the nonpathogens could reduce the $\mathrm{pH}$ to a level at which colored colonies resulted.
The mechanism by which EMB agar differentiated E. coli and Enterobacter aerogenes remained unknown. To demonstrate the mechanism of this differential action, the effect of $\mathrm{pH}$ was investigated. The molecular structure of the dye complex was determined, and a mechanism for formation of the dye complex was proposed.

\section{MATERIALS AND METHODS}

Bacterial strains and media. The strain of $E$. aerogenes was supplied by $\mathrm{M}$. M. Brent, and the culture of $E$. coli wild type was obtained from $\mathrm{S}$. Harmon, both of the Department of Biology, Bowling Green State University. The culture of $E$. coli X-961, a lactose-negative auxotroph, was supplied by R. Curtiss III of Oak Ridge Laboratories, Oak Ridge, Tenn.

Stock cultures were maintained on cystine Trypticase agar stabs (Difco Laboratories, Detroit, Mich.) at $15 \mathrm{C}$. Experimental cultures were grown at $37 \mathrm{C}$ for 18 to $24 \mathrm{~h}$ in broth containing $1 \%$ lactose, $1 \%$ peptone, and $0.2 \%$ dipotassium phosphate. EMB broth contained $0.04 \%$ eosin Y (Matheson Coleman and Bell Co., Cincinnati, Ohio) and $0.0065 \%$ methylene blue (National Aniline and Chemical Co., N.Y.) in addition to the above constituents. Solid media were prepared by adding $2 \%$ agar to the basal formula. All media were sterilized by autoclaving at $121 \mathrm{C}$ and 15 pounds pressure for $15 \mathrm{~min}$.

Methods. A Corning $\mathrm{pH}$ meter, model 7 (Corning Scientific Instruments) with Beckman pH electrodes (Beckman Co., Chicago, Ill.) was employed for $\mathrm{pH}$ determinations. The relative $\mathrm{pH}$ produced by each organism on solid media was determined by use of the indicator bromothymol blue. The medium contained 
$0.0016 \%$ bromothymol blue, $1 \%$ peptone, $0.2 \%$ dipotassium phosphate, $2 \%$ agar, and either $1 \%$ lactose, $1 \%$ glucose, or no sugar. The color of the dye at the end of $24 \mathrm{~h}$ of growth at $37 \mathrm{C}$ served as an indication of final $\mathrm{pH}$. The effect of $\mathrm{pH}$ on formation of the dye complex was investigated by employing buffer concentrations of $0.0,0.2,0.4,0.5,0.6,0.7$, $0.8,0.9$, and $1.0 \%$ in the EMB medium. All solutions were examined visually for the presence of a precipitate, and the $\mathrm{pH}$ was determined as above. The $\mathrm{pH}$ necessary to complex the dyes was also determined by titration of EMB broth with $0.01 \mathrm{~N} \mathrm{HCl}$.

The molecular weight of the dye complex was determined by the molal freezing point depression (5) using $d$-camphor as the solvent. The dye complex was prepared by reducing the $\mathrm{pH}$ to 3.5 with $1.0 \mathrm{~N} \mathrm{HCl}$. The precipitate was harvested by centrifugation at $10,000 \times g$ for $10 \mathrm{~min}$ and washed 10 times with distilled water to remove traces of unreacted eosin and methylene blue. For determination of the freezing point depression, a Buchi melting point instrument (Rinco Instrument Co., Greenville) was employed.

Ultraviolet spectra of the complex, eosin methylene blue, and a noncomplexed eosin-methylene blue solution were determined using a Beckman DB-G spectrophotometer (Beckman Co., Chicago, Ill.). The infrared spectrum of the complex was examined as a $\mathrm{KBr}$ macropellet with a Perkin-Elmer 337 doublebeam spectrophotometer (Perkin-Elmer Co., Norwalk, Conn.), as were the spectra of eosin and methylene blue.

\section{RESULTS AND DISCUSSION}

Because the differentiation of pathogenic microorganisms from nonpathogens by EMB agar was dependent on the amounts of acidic products resulting from the fermentation of lactose (7), it appeared that this mechanism might also account for the differentiation of $E$. coli and E. aerogenes. Results obtained with bromothymol blue as an indicator of final $\mathrm{pH}$ of the culture medium indicated that $E$. coli did produce a strongly acidic condition during growth at the expense of lactose, whereas $E$. aerogenes yielded a much less acidic medium. The reaction exhibited by $E$. coli X-961 was similar to that obtained with $E$. aerogenes. This observation was verified by measurement of $\mathrm{pH}$ after growth of $E$. coli and $E$. aerogenes in lactose broth. E. coli reduced the $\mathrm{pH}$ of this medium from the initial $\mathrm{pH}$ of 7.1 to 4.9 in 24 h, whereas $E$. coli X-961 yielded a $\mathrm{pH}$ of only 6.4 , and $E$. aerogenes reduced the $\mathrm{pH}$ to 5.3 in the same time period. These results were consistent with those obtained by Wynne et al. (7) and established a quantitative difference in the final $\mathrm{pH}$ resulting from fermentation of lactose by the microbial species in question.

Titration with $0.01 \mathrm{~N} \mathrm{HCl}$ indicated that the complex formed at a $\mathrm{pH}$ of about 4.9 , thereby establishing the significance of the $\mathrm{pH}$ difference observed above.

If $\mathrm{pH}$ is the critical factor in formation of the dye complex, growth of $E$. aerogenes on unbuffered EMB agar should result in a reaction typical of $E$. coli. The effect of $\mathrm{pH}$ on formation of the dye complex was observed by varying the buffer concentration from 0.0 to $1.0 \%$. Growth of $E$. aerogenes on EMB agar containing no buffer did result in the formation of a green metallic sheen, and the $\mathrm{pH}$ of the corresponding EMB broth was found to be 4.8. This sheen was not produced by $E$. aerogenes on media containing a phosphate buffer. $E$. coli, however, was capable of producing the typical reaction at buffer concentrations up to $0.5 \%$. Thus it appeared that $\mathrm{pH}$ was the critical factor in the formation of the green sheen observed with $E$. coli on EMB agar.

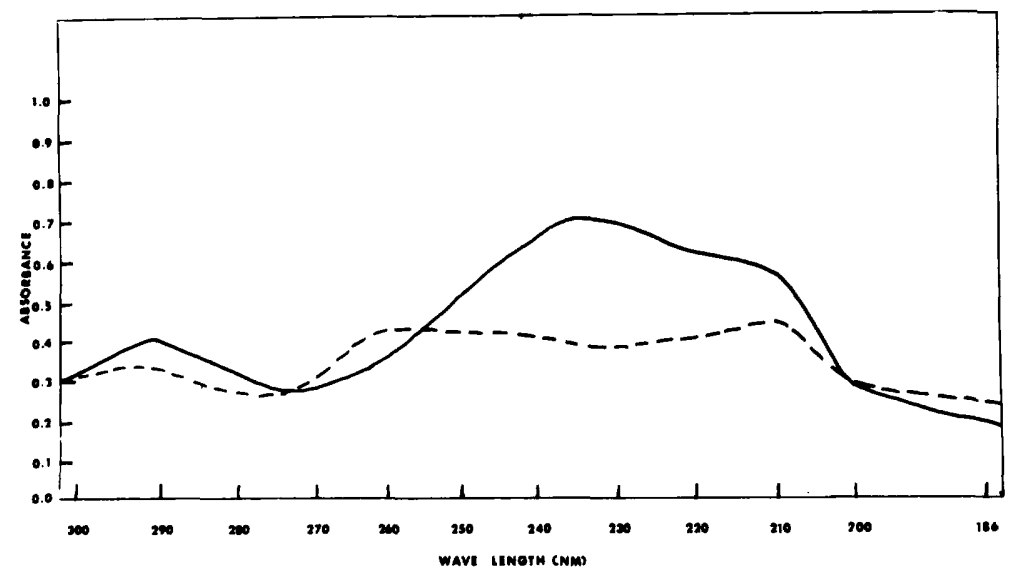

FIG. 1. Ultraviolet spectra of the dye complex (solid line) and the eosin-methylene blue uncomplexed solution (broken line). 


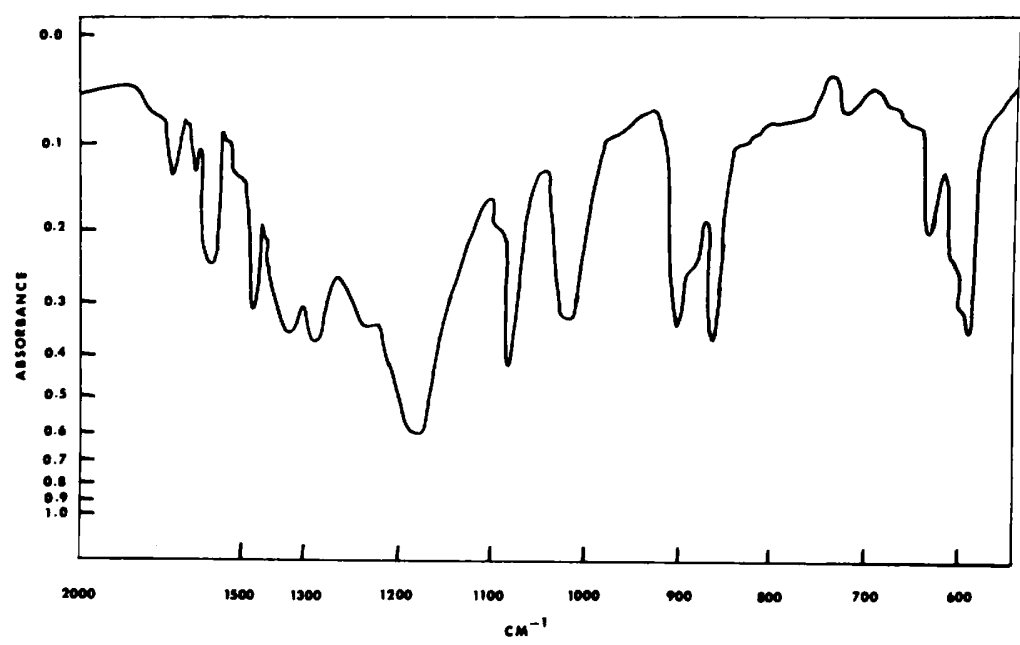

FIG. 2. Infrared spectrum of the eosin-methylene blue dye complex.

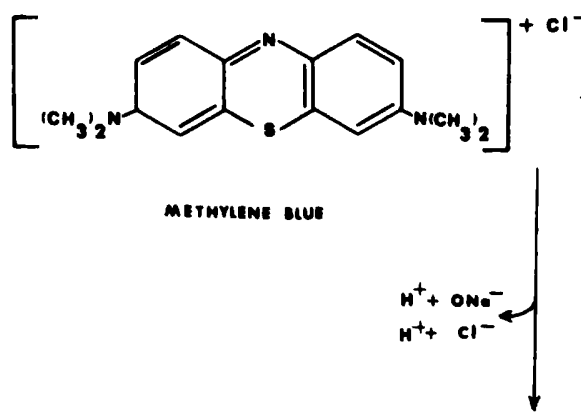

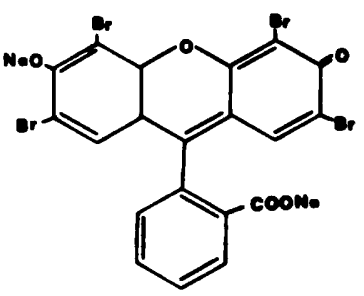

cosin

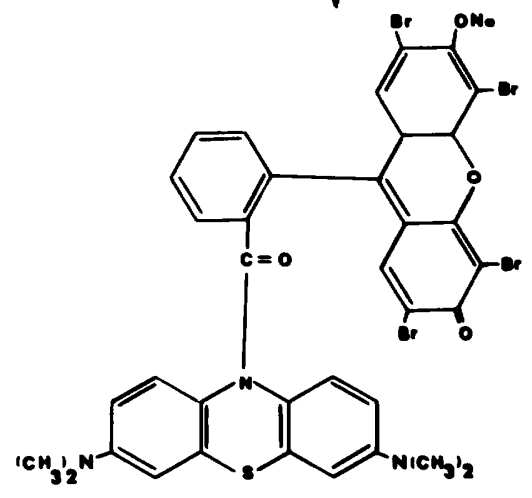

DYe complex

FIG. 3. Proposed mechanism of complexing of eosin and methylene blue under acidic conditions.

The metallic appearance of $E$. coli colonies seemed to result from the formation of a dye complex under acidic conditions. The molecular weight of this complex was determined to be 1,016 , indicating that the dyes eosin and methylene blue occurred in this complex in a ratio of 1 to 1 .
The structure of the complex was inferred from ultraviolet and infrared spectral data. As seen in Fig. 1, a significant difference in the ultraviolet spectrum of the complex was observed at $236 \mathrm{~nm}$ when compared to the spectrum of the uncomplexed solution of eosin and methylene blue. This new peak indicated 
the presence of an amide structure in the complex, and it would result from a $\mathrm{n} \rightarrow \pi *$ transition of the carbonyl group (4).

The occurrence of an amide bond in the dye complex was also indicated by an examination of the infrared spectrum of this compound (Fig. 2). Amides are characterized by a strong absorption in the 1,695 to $1,630 \mathrm{~cm}^{-1}$ region due to $\mathrm{C}=\mathrm{O}$ stretching (1). The complex exhibited a peak at $1,700 \mathrm{~cm}^{-1}$. Interpretation of this peak as indicating an amide bond was supported by a peak which occurred at 1,275 $\mathrm{cm}^{-1}$. This band would result from a $\mathrm{CNH}$ vibration in which the nitrogen and hydrogen atoms moved in the same direction relative to the carbon atom (1). A peak of $640 \mathrm{~cm}^{-1}$ was interpreted as being characteristic of an $\mathrm{NH}$ out-of-plane wag whereas a peak at $590 \mathrm{~cm}^{-1}$ was indicative of a $\mathrm{C}=\mathrm{O}$ out-of-plane bend.

Thus, the formation of the dye complex appeared to be dependent on the $\mathrm{pH}$ of the medium and involved formation of an amide bond between eosin and methylene blue, as shown in Fig. 3. The occurrence of this dye complex during growth of $E$. coli on EMB agar appeared to account for the ability of this medium to differentiate between $E$. coli and $E$. aerogenes.

\section{ACKNOWLEDGMENTS}

This work was supported by a grant from the Faculty Research Committee, Bowling Green State
University and from the National Science Foundation Institutional Committee of Bowling Green State University.

\section{REPRINT REQUESTS}

Address reprint requests to: Dr. R. S. Horvath, Department of Biology, Bowling Green State University, Bowling Green, Ohio 43403.

\section{LITERATURE CITED}

1. Colthup, N. B., L. H. Daly, and S. E. Wiberley. 1964. Introduction to infrared and raman spectroscopy, p. 263-265, 332, 382-384. Academic Press Inc., New York.

2. Holt-Harris, J. E., and O. Teague. 1916. A new culture medium for the isolation of Bacillus typhosus from stools. J. Infect. Dis, 18:596-600.

3. Levine, M. 1918. Differentiation of $B$. coli and $B$. aerogenes on a simplified eosin-methylene blue agar. J. Infect. Dis. 23:43-47.

4. Rao, C. N. R. 1967. Ultraviolet and visible spectroscopy, chemical applications, p. 193. Plenum Press, New York.

5. Schwarz, J. C. P. 1964. Physical methods in organic chemistry, p. 250-252. Holden-Day, Inc., San Francisco.

6. Wilson, T. M. 1907. Chemical and staining properties of derivatives of methylene blue and eosin. J. Exp. Med. 9:645-670.

7. Wynne, E. S., L. J. Rode, and A. E. Hayward. 1942. Mechanism of the selective action of eosin-methylene blue agar on the enteric group. Stain Technol. 17:11-20. 